\title{
Long-term outcomes of endoscopic submucosal dissection for early gastric cancer: a multicenter collaborative study
}

\author{
Satoshi Tanabe ${ }^{1} \cdot$ Kenji Ishido $^{2} \cdot$ Takayuki Matsumoto $^{3} \cdot$ Takashi Kosaka $^{3}$. \\ Ichiro Oda ${ }^{4} \cdot$ Haruhisa Suzuki $^{4} \cdot$ Junko Fujisaki $^{5} \cdot$ Hiroyuki Ono $^{6}$. \\ Noboru Kawata ${ }^{6}$. Tsuneo Oyama ${ }^{7}$. Akiko Takahashi ${ }^{7} \cdot$ Hisashi Doyama ${ }^{8}$. \\ Masaaki Kobayashi $^{9}$ - Noriya Uedo ${ }^{10} \cdot$ Kenta Hamada $^{10}$ - Takashi Toyonaga ${ }^{11}$. \\ Fumiaki Kawara $^{11} \cdot$ Shinji Tanaka $^{12}$ - Yoshikazu Yoshifuku ${ }^{12}$
}

Received: 25 August 2016/Accepted: 19 October 2016/Published online: 2 November 2016

(c) The International Gastric Cancer Association and The Japanese Gastric Cancer Association 2016

\begin{abstract}
Objective The indications for endoscopic submucosal dissection (ESD) in patients with early gastric cancer (EGC) have been expanded. However, the long-term outcomes of ESD remain unclear. We retrospectively investigated the long-term outcomes of ESD in patients with EGC.

Methods We retrospectively studied patients with EGC who underwent ESD at 11 institutions between January
\end{abstract}

This manuscript is a modified version of that published in the Japanese journal Stomach and Intestine 2014;49(11):1601-1608 in the Japanese language, entitled "Clinicopathological features, progress, and prognosis in the cases of local recurrence and metastatic recurrence following endoscopic submucosal dissection for gastric cancer: a multi-institutional joint study." Because of its important clinical implications we submitted the present English-language version of the manuscript to Gastric Cancer to reach a broader audience of readers.

Satoshi Tanabe

s-tanabe@kitasato-u.ac.jp

1 Research and Development Center for New Medical Frontiers, Kitasato University School of Medicine, Kitasato University, 1-15-1 Kitasato, Minami-ku, Sagamihara, Kanagawa 252-0374, Japan

2 Department of Gastroenterology, Kitasato University School of Medicine, Sagamihara, Japan

3 Department of Gastroenterology, Iwate Medical University, Morioka, Japan

4 Department of Endoscopy, National Cancer Center Hospital, Tokyo, Japan

5 Department of Gastroenterology, Cancer Institute Hospital of JFCR, Tokyo, Japan

6 Department of Endoscopy, Shizuoka Prefectural Cancer Center, Nagaizumi, Japan
2003 and December 2010. A total of 6456 patients (7979 lesions) who met the absolute indications for ESD and 4202 patients (5781 lesions) who met the expanded indications for ESD were studied. Clinicopathological features, clinical course, and outcomes were studied in 67 patients in whom local recurrence or metastatic recurrence was diagnosed as of March 31, 2014. The median follow-up period was 56 months.

Results Local recurrence was diagnosed in 14 patients $(0.22 \%)$ who met the absolute indications and 53 patients $(1.26 \%)$ who met the expanded indications. The rate of local recurrence was significantly higher in patients with expanded-indication lesions $(p<0.05)$. As additional treatment for recurrence, most patients received endoscopic treatment. Metastatic recurrence did not develop in any patient with absolute-indication lesions, but was diagnosed in 6 patients $(0.14 \%)$ with expanded-indication lesions

7 Department of Endoscopy, Saku Central Hospital Advanced Care Center, Saku, Japan

8 Department of Gastroenterology, Ishikawa Prefectural Central Hospital, Kanazawa, Japan

9 Department of Gastroenterology and Hepatology, Uonuma Institute of Community Medicine, Niigata University Medical and Dental Hospital, Niigata, Japan

10 Department of Gastrointestinal Oncology, Osaka Medical Center for Cancer and Cardiovascular Diseases, Osaka, Japan

11 Division of Gastroenterology, Kobe University Graduate School of Medicine, Kusunoki, Japan

12 Department of Endoscopic Medicine, Hiroshima University Hospital, Hiroshima, Japan 
$(p<0.05)$. The histological type was undifferentiated mixed type in half the patients. Three patients died of primary gastric cancer.

Conclusions ESD for expanded-indication lesions of EGC is considered an effective therapy associated with an extremely low rate of metastatic recurrence on long-term follow-up. However, fully informed consent concerning the risk of metastatic recurrence should be obtained before ESD, and close postoperative follow-up is essential.

Keywords Early gastric cancer - Absolute-indication lesions - Expanded-indication lesions $\cdot$ ESD $\cdot$ Outcomes

\section{Introduction}

More than 10 years have elapsed since the development of endoscopic dissection (ESD). Improvements in instruments and devices and progress in techniques have led to the rapid dissemination of ESD in clinical practice. ESD has enabled the en bloc resection of lesions that previously had to be resected in a piecemeal fashion by conventional endoscopic mucosal resection (EMR) [1]. One of the most important features of ESD is that accurate pathological evaluation is possible.

Clinical expansion of the indications for ESD has been attempted on the basis of the findings of Gotoda et al. [2]. However, the long-term outcomes of lesions meeting the expanded indications for ESD remain unclear [3, 4]. In the present multicenter collaborative study, we retrospectively evaluated the long-term outcomes of lesions that met the absolute indications (absolute-indication lesions) or the expanded indications (expanded-indication lesions) for ESD in patients with early gastric cancer (EGC). The study protocol and analysis were approved by the ethics committees of each participating hospital.

\section{Subjects}

Among patients with EGC (absolute-indication lesions or expanded-indication lesions) who underwent ESD from January 2003 through December 2010, we studied patients who had local recurrence or metastatic recurrence as of March 31, 2014 (i.e., patients who were followed up for at least 3 years).

\section{Methods}

The study protocol was prepared, and the co-investigators requested the ethics committee in each hospital to review the study protocol. The study protocol was approved by all hospitals. Satoshi Tanabe was the chief investigator, and
Kenji Ishido was in charge of the study secretariat office, which sent the patient case report forms to the co-investigators. The responsible co-investigator at each participating hospital completed the case report forms and sent them to the study secretariat office, which then compiled and analyzed the study data. Statistical analysis was performed with chi-square tests, and $P$ values of less than 0.05 were considered to indicate statistical significance.

\section{Study variables}

The following variables were studied: age at the time of treatment, sex, concurrent underlying diseases, conditions defining indications (absolute-indication lesion or expanded-indication lesion), macroscopic type, presence or absence of ulcer, tumor location, preoperative invasion depth (mucosa, M or submucosa, SM), pathological invasion depth (mucosa, $\mathrm{M}$ or first layer of the submucosa, SM1), greatest tumor diameter, main histological type, undifferentiated mixed-type cancer (differentiated-typepredominant or undifferentiated-type-predominant), lymphovascular invasion (lymph nodes, ly or blood vessels, v), resection margins (horizontal margin, HM and vertical margin, VM), additional treatment after ESD, date of confirming local residual tumor or recurrence, treatment for local residual tumor or recurrence, presence or absence of metastatic recurrence, treatment for metastatic recurrence, outcomes, and the cause of death (death from gastric cancer, death from other diseases). Lymphatic involvement was evaluated by D2-40 immunostaining.

Criteria defining absolute-indication lesions and expanded-indication lesions according to the Japanese Gastric Cancer Association (JGCA) state absolute-indication criteria for the endoscopic treatment of differentiated mucosal cancer include tumors less than $2 \mathrm{~cm}$ in diameter, without ulceration [5]. On the other hand, the expanded-indication criteria proposed by Gotoda et al. [2] recommend endoscopic resection for the management of differentiated mucosal cancer regardless of size in the absence of ulceration or tumors less than $3 \mathrm{~cm}$ in diameter in the presence of ulceration. In addition, we included lesions with minute submucosal invasion (SM1: invasion depth $500 \mu \mathrm{m}$ below the muscularis mucosae) that were less than $30 \mathrm{~mm}$ in diameter.

\section{Results}

\section{Subjects (Table 1)}

The study group comprised patients with EGC who underwent ESD in the 11 participating hospitals from January 2003 through December 2010. A total of 6456 
Table 1 Patient registration and analysis

\begin{tabular}{lll}
\hline & Absolute indication & Expanded indication \\
\hline No. of patients & 6456 & 4202 \\
No. of lesions & 7979 & 5781 \\
Local recurrence & $14(0.22 \%)^{*}$ & $53(1.26 \%)^{*}$ \\
Metastatic recurrence & $0(0 \%)^{*}$ & $6(0.14 \%)^{*}$ \\
\hline
\end{tabular}

$* p<0.05$ ( $\chi^{2}$ analysis $)$

patients with 7979 absolute-indication lesions and 4202 patients with 5781 expanded-indication lesions were studied. The median follow-up was 56 months (range, 39-76 months). The overall capture rate was $76.0 \%$. Outcomes were confirmed by telephone or letter in about $50 \%$ of patients with each type of lesion.

\section{Local recurrence rates (Table 1)}

Local recurrence occurred in 14 patients $(0.22 \%)$ with absolute-indication lesions and 53 patients $(1.26 \%)$ with expanded-indication lesions. The rate of local recurrence was significantly higher in patients with expanded-indication lesions $(p<0.05)$.

\section{Clinicopathological features of local recurrence (Table 2)}

The sites of local residual tumor or recurrence were approximately equally distributed in the upper, middle, and lower thirds of the stomach for both absolute-indication lesions and expanded-indication lesions. Macroscopically, the majority of absolute-indication lesions and expandedindication lesions were flat or depressed. The median tumor diameter was significantly greater for expanded-indication lesions $(30 \mathrm{~mm})$ than for absolute-indication lesions $(7 \mathrm{~mm})$. The depth of tumor invasion was intramucosal (M) in 49 of the 53 patients with expanded-indication lesions. The histological type was undifferentiated mixedtype cancer in $1(7 \%)$ of the 14 patients with local recurrence of absolute-indication lesions and $9(17 \%)$ of the 53 patients with local recurrence of expanded-indication lesions (differentiated-type predominant in 7 patients and undifferentiated-type predominant in 2 patients). The horizontal margin was positive in $7(50 \%)$ patients with absolute-indication lesions and $30(57 \%)$ patients with expanded-indication lesions. The vertical margin was positive in no patient with absolute-indication lesions and in 1 patient with expanded-indication lesions. The vertical margin could not be evaluated in 5 patients because of heat denaturation. The median time to the development of local recurrence was significantly shorter in patients with expanded-indication lesions ( 8 months) than in those with absolute-indication lesions (15 months).

\section{Courses and outcomes of patients with local recurrence}

\section{Absolute-indication lesions (Fig. 1)}

Additional treatment for the 14 patients who had local recurrence of absolute-indication lesions comprised endoscopic treatment in 12 patients and surgery in 1 patient. The other patient was followed up with no further treatment because of advanced age. The endoscopic treatment procedure was ESD in 11 patients and argon plasma coagulation (APC) in 1. At the time of this writing, all these patients are still alive with no subsequent recurrence. The patient who was followed up without additional treatment died of another disease.

\section{Expanded-indication lesions (Fig. 2)}

The clinical courses of the 53 patients who had local recurrence of expanded-indication lesions are shown in
Table 2 Clinicopathological features of local recurrence in patients with absolute- and expanded-indication lesions

\begin{tabular}{llll}
\hline No. of patients & 14 (absolute) & 53 (expanded) & $p$ \\
\hline Location: upper third/middle third/lower third & $4 / 6 / 4$ & $14 / 18 / 21$ & 0.73 \\
Macroscopic type: elevated/flat/depressed & $5 / 2 / 7$ & $20 / 3 / 30$ & 0.54 \\
Median tumor size (range) & $7 \mathrm{~mm}(5-20)$ & $30 \mathrm{~mm}(10-95)$ & 0.00 \\
Tumor depth, M/SM1 & $14 / 0$ & $49 / 4$ & 0.28 \\
Types of histology & & \\
Diff/mixed (diff $>$ un)/mixed(un $>$ diff)/un & $13 / 1 / 0 / 0$ & $44 / 7 / 2 / 0$ & 0.60 \\
Horizontal margin, positive/negative/ne & $7 / 5 / 2$ & $30 / 13 / 10$ & 0.69 \\
Vertical margin, positive/negative/ne & $0 / 14 / 0$ & $1 / 47 / 5$ & 0.41 \\
Curability, curative/noncurative & $5 / 9$ & $13 / 40$ & 0.40 \\
Median time to local recurrence (range) & 15 months (2-84) & 8 months (1-87) & 0.03 \\
\hline diff differentiated, mixed differentiated and undifferentiated, un undifferentiated, ne not evaluated
\end{tabular}




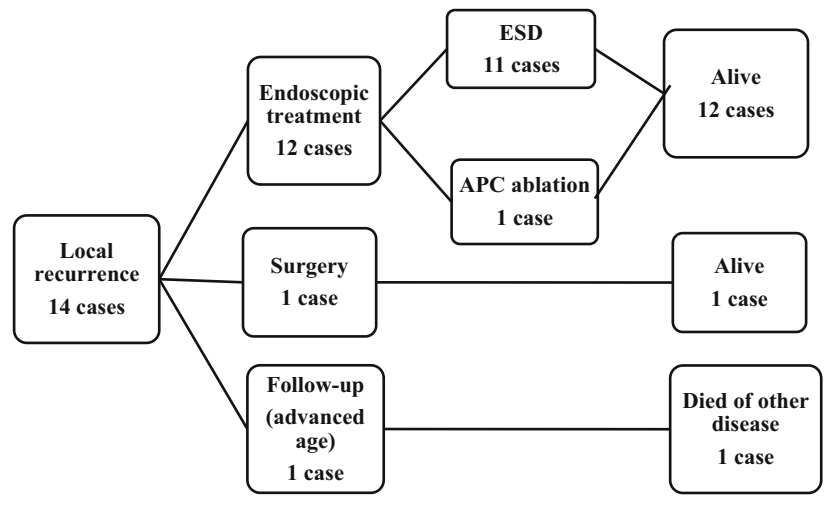

APC: argon plasma coagulation

Fig. 1 Clinical course of local recurrence in patients with absoluteindication lesions $(n=14)$. ESD endoscopic submucosal dissection, $A P C$ argon plasma coagulation

Fig. 2. Additional treatment for local recurrence comprised endoscopic treatment in 46 patients, surgery in 5 patients, and chemotherapy in 1 patient who had local as well as metastatic recurrence. Only 1 patient was followed up with no further treatment. The procedure used in the 46 patients who received endoscopic treatment was EMR in 1 patient, ESD in 41 patients, APC in 2 patients, photodynamic therapy (PDT) in 1 patient, and coagulation with a "hot biopsy" forceps in 1 patient. ESD was thus performed in nearly $90 \%$ of the patients who received additional endoscopic treatment. Table 3 summarizes the characteristics of the 5 patients who underwent surgery for local recurrence of expanded-indication lesions. Three patients had differentiated-type mucosal cancer exceeding $3 \mathrm{~cm}$ in diameter, 1 patient had differentiated-type mucosal cancer measuring $3 \mathrm{~cm}$ or less in diameter and accompanied by an ulcer, and 1 patient had differentiated-type SM1 cancer measuring less than $3 \mathrm{~cm}$ in diameter. Three of the 5 patients had undifferentiated mixed-type cancer. All patients who underwent surgical resection are alive. All 6 patients who died after endoscopic treatment died of other diseases.

\section{Clinicopathological characteristics of patients with metastatic recurrence}

Metastatic recurrence occurred in six patients $(0.14 \%)$ with expanded-indication lesions and in no patient with absolute-indication lesions $(p<0.005)$ (Table 1$)$. The characteristics of the six patients with metastatic recurrence are summarized in Table 4. Age range was 57 to 77 years, and five of the patients were men. The depth of invasion was intramucosal in three patients and SM1 in the three other patients. The tumor diameter was less than about $20 \mathrm{~mm}$, except for one patient with a tumor diameter of $55 \mathrm{~mm}$. The histological type was differentiated-type-predominant, undifferentiated mixed-type cancer in half the patients. All lateral and vertical resection margins were tumor negative, and resection was evaluated to be curative in all patients. Only one patient had local recurrence accompanied by metastatic recurrence. The time to metastatic recurrence ranged from 16 to 87 months (median, 50 months) and was 50 months or longer in four patients. The site of metastatic
Fig. 2 Clinical course of local recurrence in patients with expanded-indication lesions $(n=53)$. EMR endoscopic mucosal resection, $A P C$ argon plasma coagulation, $P D T$ photodynamic therapy, HOT hot biopsy

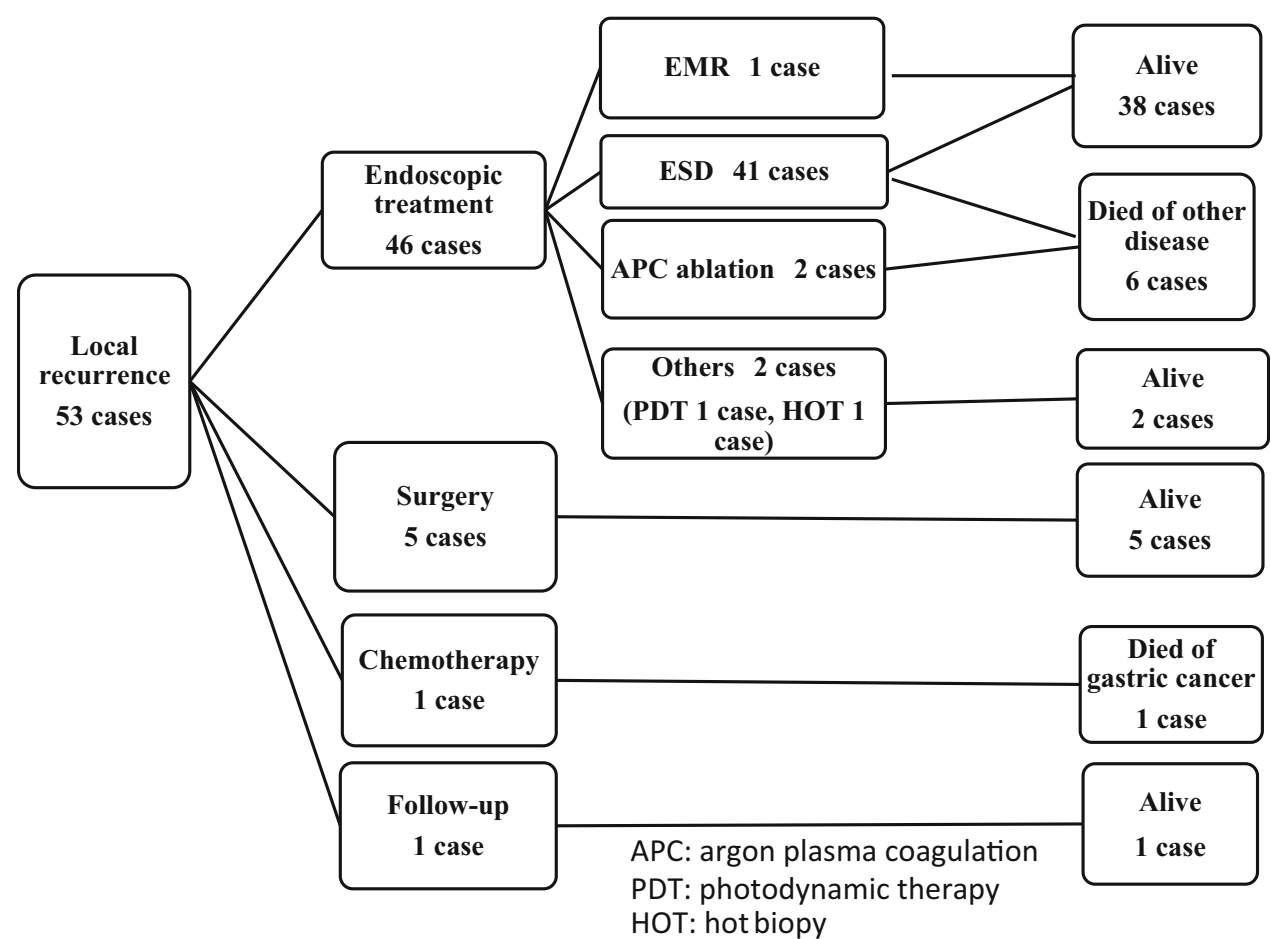


Table 3 Characteristics of patients with local recurrence of expanded-indication lesions who underwent additional surgery

\begin{tabular}{|c|c|c|c|c|c|c|c|c|c|c|c|c|}
\hline & Age & Sex & $\begin{array}{l}\text { Macroscopic } \\
\text { type }\end{array}$ & Ulcer & Depth & Tumor size & Histology & $\mathrm{HM}$ & VM & Curability & $\begin{array}{l}\text { Time to } \\
\text { recurrence } \\
\text { (months) }\end{array}$ & Outcome \\
\hline 1 & 74 & $\mathrm{M}$ & 0-IIc & + & M & 30 & tub2>por-sig & - & - & Curative & 33 & Alive \\
\hline 2 & 51 & $\mathrm{~F}$ & 0-IIc & - & M & 45 & $\begin{array}{l}\text { tub1>tub2>por, } \\
\text { sig }\end{array}$ & + & - & Non-curative & 1 & Alive \\
\hline 3 & 70 & $\mathrm{~F}$ & 0-IIa & - & M & 50 & tub1 $>$ tub2 & + & - & Non-curative & 16 & Alive \\
\hline 4 & 49 & $\mathrm{M}$ & 0-IIa+IIc & - & M & 50 & tub2>por & ne & - & Non-curative & 12 & Alive \\
\hline 5 & 80 & M & 0-IIc & + & SM1 & 9 & tub1 & + & - & Non-curative & 4 & Alive \\
\hline
\end{tabular}

Tub1 well differentiated adenocarcinoma, Tub2 moderately differentiated adenocarcinoma, ne not evaluated, por poorly differentiated adenocarcinoma, sig signet ring cell carcinoma

recurrence was lymph nodes in five patients and the lung in one. As additional treatment, two patients underwent surgery and three received chemotherapy. The remaining patient was followed up with no further treatment. At the time of this writing, three of the six patients are still alive, and three have died of gastric cancer.

\section{Discussion}

The advent of ESD has led to substantial progress in endoscopic treatment for EGC. The indication range of ESD is now being broadened from absolute-indication lesions to include expanded-indication lesions. The longterm outcomes of ESD in patients with absolute-indication lesions have been reported to be equivalent to those of curative surgery [6]. However, a consensus has yet to be reached concerning the long-term outcomes of ESD in patients with expanded-indication lesions [3, 4, 7]. In clinical practice, treatment is performed after patients are informed that the indication range of ESD is currently being expanded on a clinical research basis.

Local recurrence and metastatic recurrence have different underlying mechanisms. Local recurrence can occur when curative (R0) resection is not achieved at lateral or deep margins. In contrast, metastatic recurrence is associated with the presence of risk factors such as submucosal microinvasion and lymphovascular invasion even after R0 resection. The rate of locoregional recurrence has been reported to range from $1.9 \%$ to $6.6 \%$ for conventional EMR [8-11], as compared with only $0.2 \%$ to $3.7 \%$ for ESD with en bloc resection [12-14]. In our previous singlecenter retrospective study [12], the rate of local recurrence of absolute-indication lesions was significantly lower in patients who underwent ESD $(0 \%)$ than in those who underwent EMR (2.9\%). In patients with expanded-indication lesions, the rate of local recurrence was also significantly lower for ESD (0.6\%) than for EMR (12.5\%). These findings showed that the local recurrence rate of expanded-indication lesions was particularly high after EMR and clearly demonstrated the value of en bloc resection by ESD.

In the present multicenter collaborative study, we analyzed local recurrence and metastatic recurrence in a series of more than 10,000 patients with EGC who underwent ESD. The local recurrence rate was significantly higher for expanded-indication lesions than for absolute-indication lesions.

As for the clinicopathological characteristics associated with local recurrence of expanded-indication lesions, the most common macroscopic type of tumor was flat or depressed type, which was present in $75.5 \%$ of the patients who underwent noncurative resection. Several groups of investigators have reported that incomplete resection and tumor-positive horizontal resection margins are risk factors for local recurrence [13-16]. Imagawa et al. [17] reported that tumor location (upper third of the stomach) and size $(\geq 2 \mathrm{~cm})$ are technical factors related to incomplete resection. Takenaka et al. [13] also proposed that tumor location in the upper part of the stomach and a tumor diameter $\geq 30 \mathrm{~mm}$ are risk factors for local recurrence. Kakushima et al. [18] reported that a tumor diameter $\geq 30 \mathrm{~mm}$, recurrent lesions, submucosal invasion, and undifferentiated cancer are risk factors for a positive horizontal resection margin. In our study, the median tumor diameter in patients who had local recurrence of expanded-indication lesions was $30 \mathrm{~mm}$. Han et al. [19] studied the relationship between histological type and local recurrence and found that the local recurrence rate was significantly higher in patients with histologically mixed-type cancer $(19.8 \%)$ than in those with differentiated-type cancer $(1.7 \%)$ or undifferentiated-type cancer $(9.8 \%)$. Their analysis indicated that histologically mixed-type cancer is associated with a higher rate of incomplete resection. Because 9 patients with local recurrence of expanded-indication lesions in our series had undifferentiated mixed-type cancer, preoperative diagnosis might have been challenging. A recent study 


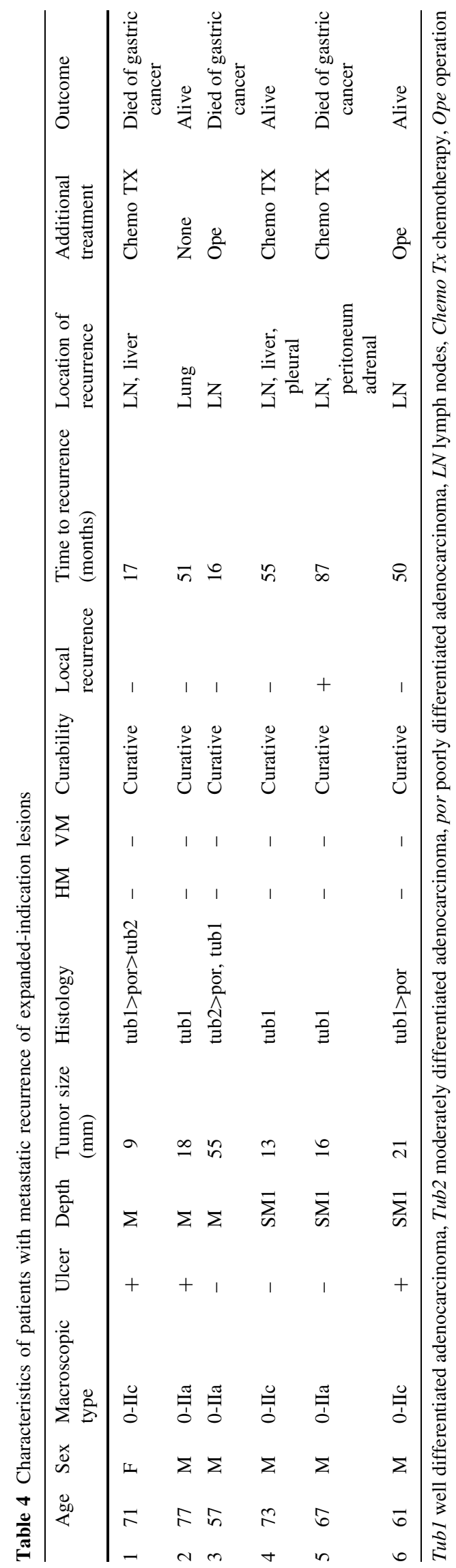

has reported the usefulness of image-enhanced endoscopy and magnifying endoscopy [20]. However, these endoscopic techniques have their limitations in patients with undifferentiated mixed-type cancer [21], and other measures, including preoperative biopsy of the surrounding region, are most likely required for an accurate diagnosis. Sekiguchi et al. [22] studied local recurrence in patients with tumor-positive horizontal margins and reported that a positive horizontal margin of $6 \mathrm{~mm}$ or longer is an independent risk factor for local recurrence. How to treat patients with positive horizontal margins remains a matter of debate. Various treatments are currently used on a patient-specific and hospital-specific basis.

Endoscopic treatment was used to manage local recurrence in $12(86 \%)$ of 14 patients with absolute-indication lesions, 11 of whom underwent ESD, and in $46(87 \%)$ of 53 patients with expanded-indication lesions, 41 of whom underwent ESD. ESD has been reported to be useful for the treatment of local recurrence [23], but it is associated with the development of fibrous scar tissue, a high level of technical difficulty, and a high risk of perforation. These problems should be addressed in future studies.

Metastatic recurrence was not found in any patient with absolute-indication lesions but developed in six patients with expanded-indication lesions $(0.14 \%)$. This difference was statistically significant. Several previous studies have evaluated outcomes in patients with expanded-indication lesions and found no difference in outcomes between expanded-indication lesions and absolute-indication lesions [3, 4, 7]. All these studies were conducted in a single center and had a relatively small sample size. In our study, only a very few patients had metastatic recurrence. We believe that patients with expanded-indication lesions should be followed up according to a stricter protocol than patients with absolute-indication lesions.

The theoretical basis for expanding the indication range of ESD is as follows. In the National Cancer Center Hospital, the 5-year survival rate after surgical resection of EGC is $99 \%$ in patients with mucosal cancer and $96 \%$ in those with submucosal cancer, excluding deaths from other diseases.

Therefore, if the rate of metastasis after ESD was $1 \%$ or less for mucosal cancer and $4 \%$ or less for submucosal cancer, the outcomes of ESD were hypothesized to be equivalent to those of surgical resection. On the basis of this theoretical calculation, the rate of metastatic recurrence in our study was within expectation. As for the risk of surgery, Kikuchi et al. [24] reported that $3(0.35 \%)$ of 851 patients with EGC died of surgery-related causes. Surgery is therefore associated with a certain risk. Given that all confirmed cases of metastatic recurrence in our study developed in patients who were evaluated to have undergone curative resection, the risk of metastatic recurrence 
after endoscopic treatment for expanded-indication lesions should be thoroughly explained to patients and informed consent should be obtained preoperatively, even though the risk is less than $1 \%$. To our knowledge, this is the first multicenter study to evaluate the risk of metastatic recurrence after ESD in such a large series of patients with EGC. We therefore consider our results valuable.

As for ESD for expanded-indication lesions of early gastric cancer, ulceration and submucosal invasion have been reported to be risk factors for metastatic recurrence [2]. Undifferentiated mixed-type cancer has been reported to be associated with higher biological malignancy than pure differentiated-type cancer, potentially leading to high rates of lymphatic invasion and lymph node metastasis [25]. Takizawa et al. [26] studied intramucosal gastric cancer in patients who underwent surgery and reported that $13.2 \%$ of patients had mixed predominantly differentiated cancer (MD) and $10.2 \%$ had mixed predominantly undifferentiated cancer (MU). The rate of lymph node metastasis was high in patients with MU (24\%). In contrast, none of the patients with MD had lymph node metastasis. In our study, three of the six patients with metastatic recurrence had undifferentiated mixed-type gastric cancer. The management of such cases, including preoperative diagnosis, is an issue for future studies.

Our study had several important limitations. It had a retrospective design and mainly evaluated patients who had recurrence. There was no matching between patients with absolute-indication lesions and those with expanded-indication lesions, and the ESD technique and follow-up protocol were not standardized. The results of a prospective clinical study by the Japan Clinical Oncology Group (JCOG0607 study) [27] are awaited.

In conclusion, metastatic recurrence was not observed in any patient with absolute-indication lesions but did develop in a very small number of patients with expanded-indication lesions. These results are considered equivalent to those of surgical therapy. ESD for expanded-indication lesions of early gastric cancer is associated with an extremely low rate of metastatic recurrence on long-term follow-up and is thus considered an appropriate treatment. However, fully informed consent about the risk of metastatic recurrence should be obtained before ESD, and close postoperative follow-up is essential.

\section{Compliance with ethical standards}

Conflict of interest The authors declare that they have no conflict of interest.

Human rights statement All procedures followed were in accordance with the ethical standards of the responsible committee on human experimentation (institutional and national) and with the Helsinki Declaration of 1964 and later versions.

\section{References}

1. Tanabe S, Koizumi W, Mitomi H, Nakai H, Murakami S, Nagaba $\mathrm{S}$, et al. Clinical outcome of endoscopic aspiration mucosectomy for early stage gastric cancer. Gastrointest Endosc. 2002;56(5):708-13.

2. Gotoda T, Sasako M, Yanagisawa A, Ono H, Nakanishi Y, Shimoda $\mathrm{T}$, et al. Incidence of lymph node metastasis from early gastric cancer; estimation with a large number of cases at two large centers. Gastric Cancer. 2000;3:219-25.

3. Gotoda T, Iwasaki M, Kusano C, Seewald S, Oda I. Endoscopic resection of early gastric cancer treated by guideline and expanded National Cancer Center criteria. $\mathrm{Br}$ J Surg. 2010;97(6):868-71.

4. Yamaguchi N, Isomoto H, Fukuda E, Ikeda K, Nishiyama H, Akiyama M, et al. Clinical outcomes of endoscopic submucosal dissection for early gastric cancer by indication criteria. Digestion. 2009;80(3):173-81.

5. Japanese Gastric Cancer Association. Japanese gastric cancer treatment guidelines 2014 (ver. 4). Gastric Cancer 2016 (published online).

6. Uedo N, Iishi H, Tatsuta M, Ishihara R, Higashino K, Takeuchi $\mathrm{Y}$, et al. Long term outcomes after endoscopic mucosal resection for early gastric cancer. Gastric Cancer. 2006;9:88-92.

7. Goto O, Fujishiro M, Kodashima S, Ono S, Omata M. Outcomes of endoscopic submucosal dissection for early gastric cancer with special reference to validation for curability criteria. Endoscopy. 2009;41(2):118-22.

8. Ono H, Kondo H, Gotoda T, Shirao K, Yamaguchi H, Saito D, et al. Endoscopic mucosal resection for treatment of early gastric cancer. Gut. 2001;48:225-9.

9. Kojima T, Parra-Blanco A, Takahashi H, Fujita R. Outcome of endoscopic mucosal resection for early gastric cancer: review of the Japanese literature. Gastrointest Endosc. 1998;48:550-4.

10. Oda I, Saito D, Tada M, Iishi H, Tanabe S, Oyama T, et al. A multicenter retrospective study of endoscopic resection for early gastric cancer. Gastric Cancer. 2006;9:262-70.

11. Oka S, Tanaka S, Kaneko I, Mouri R, Hirata M, Kawamura T, et al. Advantage of endoscopic submucosal dissection compared with EMR for early gastric cancer. Gastrointest Endosc. 2006;64:877-83.

12. Tanabe S, Ishido K, Higuchi K, Sasaki T, Katada C, Azuma M, et al. Long-term outcome of endoscopic submucosal dissection for early gastric cancer. A retrospective comparison with conventional endoscopic resection in a single center. Gastric Cancer. 2014;17:130-6.

13. Takenaka R, Kawahara Y, Okada H, Hori K, Inoue M, Kuwano $\mathrm{S}$, et al. Risk factors associated with local recurrence of early gastric cancers after endoscopic submucosal resection. Gastrointest Endosc. 2008;68:887-94.

14. Park JC, Lee SK, Seo JH, Kim YJ, Chung H, Shin SK, et al. Predictive factors for local recurrence after endoscopic resection for early gastric cancer: long-term clinical outcome in a singlecenter experience. Surg Endosc. 2010;24:2842-9.

15. Jung H, Bae JM, Choi MG, Noh JH, Sohn TS, Kim S. Surgical outcome after incomplete endoscopic submucosal dissection of gastric cancer. Br J Surg. 2011;98:73-8.

16. Yoon H, Kim SG, Choi J, Im JP, Kim JS, Kim WH, et al. Risk factors of residual or recurrent tumor in patients with a tumorpositive resection margin after endoscopic resection of early gastric cancer. Surg Endosc. 2013;27:1561-8. 
17. Imagawa A, Okada H, Kawahara $\mathrm{Y}$, Takenaka R, Kato J, Kawamoto H, et al. Endoscopic submucosal dissection (ESD) for early gastric cancer: results and degrees of technical difficulty as well as success. Endoscopy. 2006;38:987-90.

18. Kakushima N, Ono H, Tanaka M, Takizawa K, Yamaguchi Y, Matsubayashi $\mathrm{H}$. Factors related to lateral margin positivity for cancer in gastric specimens of endoscopic submucosal dissection. Dig Endoscopy. 2011;23:227-32.

19. Han JP, Hong SJ, Kim HK. Long-term outcomes of early gastric cancer diagnosed as mixed adenocarcinoma after endoscopic submucosal dissection. J Gastroenterol Hepatol. 2015;30(2):316-20.

20. Sumiyama K, Kaise M, Nakayoshi T, Kato M, Mashiko T, Uchiyama $\mathrm{Y}$, et al. Combined use of a magnifying endoscope with a narrow band imaging system and multibending endoscope for en bloc EMR of early stage gastric cancer. Gastrointest Endosc. 2004;60:79-84.

21. Oyama T, Takahashi A, Tomori A, Shinohara T, Kishino T, Takeda S, et al. Magnified endoscopic findings of early gastric cancers mixed with well and poorly differentiated type. Stomach Intest (Tokyo). 2013;48:1619-28.

22. Sekiguchi M, Suzuki H, Oda I, Abe S, Nonaka S, Yoshinaga S, et al. Risk of recurrent gastric cancer after endoscopic resection with a positive lateral margin. Endoscopy. 2014;6:273-8.
23. Sekiguchi M, Suzuki H, Oda I, Abe S, Nonaka S, Yoshinaga S, et al. Favorable long-term outcomes of endoscopic submucosal dissection for locally recurrent early gastric cancer after endoscopic resection. Endoscopy. 2013;45:708-13.

24. Kikuchi S, Katada N, Sakuramoto S, Kobayashi N, Shimao H, Watanabe M, et al. Survival after surgical treatment of early gastric cancer: surgical techniques and long-term survival. Arch Surg. 2004;389:69-74.

25. Hanaoka N, Tanabe S, Mikami T, Okayasu I, Saigenji K. Mixedhistologic-type submucosal invasive gastric cancer as a risk factor for lymph node metastasis: feasibility of endoscopic submucosal dissection. Endoscopy. 2009;41:427-32.

26. Takizawa K, Ono H, Kakushima N, Tanaka M, Hasuike N, Matsubayashi $\mathrm{H}$, et al. Risk of lymph node metastases from intramucosal gastric cancer in relation to histological types: how to manage the mixed histological type for endoscopic submucosal dissection. Gastric Cancer. 2013;16:531-6.

27. Kurokawa Y, Hasuike N, Ono H, Boku N, Fukuda H. A phase II trial of endoscopic submucosal dissection for mucosal gastric cancer: Japan Clinical Oncology Group Study JCOG0607. Jpn J Clin Oncol. 2009;39(7):464-6. 